\title{
Online Academic Dishonesty of College Students: A Review
}

\author{
Changsheng Chen ${ }^{1}$, Jingyun Long ${ }^{2,}{ }^{*}$, Junxiao Liu ${ }^{3}$, Zongjun Wang ${ }^{1}$, Lifeng Wang ${ }^{1}$, \\ Jing Zhang ${ }^{1}$
}

\author{
${ }^{1}$ Shandong Youth University of Political Science, Jinan 250103, China \\ ${ }^{2}$ Long Ao School of Jinan, Jinan 250015, China \\ ${ }^{3}$ Library, Shandong Normal University, Jinan 250014, China \\ *Corresponding author
}

\begin{abstract}
e-Learning provides students with an open, free, and self-supervised scene, which has found a feasible path for lifelong education. The quality of online learning and the certification of achievements must be based on the real learning process. According to the existing literature, research on online academic dishonesty (OAD) is relatively rare. We need to advance future research based on the past experience on online academic dishonesty, and we also need to systematically sort out the research conclusions and possible development directions of this topic. In this paper, the author systematically combed the relevant literature on online academic dishonesty, covering most of the relevant papers as of the end of 2019. Most research on online academic fraud has the following characteristics: using questionnaires to obtain exploration conclusions, focusing on learners in a certain discipline or situation, lack of research on big data learning behavior sample data, and the formation mechanism of online academic dishonesty behavior needs to be further studied and elaboration. The research results show that online academic dishonesty is indeed widespread, and the factors that cause the dishonesty behavior come from many aspects such as personality, cognition, teaching, system, etc. In future research, it is necessary to identify online academic dishonesty in time and take comprehensive intervention measures to make integrity and moral culture in online environments.
\end{abstract}

Keywords: Online academic dishonesty, e-Dishonesty, Lifelong learning, Literature review

\section{INTRODUCTION}

At present, all countries in the world will strive to promote the widespread application of online open courses, build online open courses suitable for online communication and teaching activities with high content quality and good teaching results, and explore online learning certification and credit recognition mechanisms. As an information technology, online teaching is revolutionizing traditional teaching methods. Online learning has played an active role in promoting the reform of university curriculum teaching, but its inherent unsupervised and self-paced characteristics have triggered a series of online education quality crises. Online academic dishonesty is a kind of problem-learning behavior with low immersion and lack of integrity, and many studies have confirmed that there is a large number of students' online academic dishonesty. Pavela (1997) proposed a framework of types of dishonesty based on academic dishonesty scenarios, namely cheating, plagiarism, forgery, and convenience (for others' academic dishonesty) [1]. King et al. (2009) believe that cheating is the learner's fault against academic integrity, as well as the unfair advantage and misrepresentation of knowledge [2]. Raines et al. (2011) researched the online learning environment and proposed that online classroom cheating is a violation of university policies and benefits from individuals or others, rather than relying on their own ability to obtain academic results [3]. To curb the problem of dishonesty in online education, the United States introduced the Higher Education Opportunity Act (2008) [4]. The research believes that if the problem of mistrust of online learning cannot be effectively solved, it will not only seriously affect the quality of online learning and certification of results [5] and the effectiveness of integrity education in universities, but also seriously damage national education investment and people's expectations, and affect education modernization quality. Therefore, in order to build a national integrity system and achieve the goals of education reform, we must attach great importance to the transformative role of online courses in teaching and learning, and in-depth study of the formation mechanism and prevention and control mechanism of learners' online academic dishonesty has become a key science problem that needs urgent solution. 


\section{RESEARCH QUESTIONS}

The purpose of this study is to review the existing literature on online learning of dishonesty, to focus on the concerns of previous studies, the new perspectives and findings of major researchers, and to propose the direction of future research in this field. In this section, we propose the goals of this study and related research questions.

RQ1: What are the characteristics and patterns of college students' learning dishonesty behavior in online learning?

Compared with ordinary learning behavior, dishonesty learning behavior is a special behavior manifestation in learners' online learning. The dishonesty learning behavior has special behavior motivation, stimulating process and behavior model, which will have a special impact on the academic development and future professional development of the learner. From the perspective of social learning, the widespread existence of student dishonesty learning behaviors will have a negative impact on the learning behaviors of more students. Therefore, it is necessary to have a deep understanding of the characteristics and behavior patterns of students' academic dishonesty behavior in the online learning environment.

RQ2: What are the factors that cause college students to learn dishonesty in online learning?

Previous researchers have paid more attention to learning dishonesty in non-digital environments, and put forward some factors that stimulate learners to produce dishonesty. However, how can the digital learning environment with advantages of openness, flexibility, and self-supervision become a space where dishonest behavior prevails. What causes it? What are the factors that make it develop and spread? This is a problem that researchers generally care about, and it is also a difficult problem.

RQ3: What are the methods to identify college students' academic dishonesty behavior in online learning?

Unlike classrooms where teachers and students are in the same scene, in the online learning environment, after completing the curriculum design and publishing, teachers often only synchronize with students in the forum Q \& A session. Online learners are often in a low presence, as a result, College students' behavior online academic dishonesty is often hidden, which is very difficult to find and identify. Effectively identifying the dishonest behavior of college students in online learning is a prerequisite for correcting or reducing the occurrence of dishonesty. Fortunately, the learning management system(LMS) records relatively complete user behavior information, which provides data support for interfering with students' dishonest behavior.

RQ4: What strategies can be adopted to correct or reduce the occurrence of college students' learning dishonesty?

Online courses represented by large-scale online open courses are being integrated into classroom teaching in various ways. Educational administrations in various countries look forward to using online courses as a basic environment for lifelong education with high hopes. With the in-depth application of online courses, it is bound to face the problems of learning quality assurance and identification of learning results. Therefore, it is necessary to promptly and effectively correct or reduce the academic dishonesty of college students. At present, what the researchers put forward, what attempts they have made, and how effective they are the focus of this study.

\section{METHODOLOGY}

\subsection{Planning Phase}

This paper focuses on a systematic literature review of online academic dishonesty. The research steps include planning, conducting and reporting [6]. This section mainly introduces the planning and conducting phases, and the research results will be reported in the next section.

In order to fully display the latest developments in the current study of online academic dishonesty this study mainly selects Google Scholar and CNKI as the literature search database. There is no time limit or category setting in the literature selection. Using "learning dishonesty", "learning dishonesty", "exam plagiarism" and "scholastic deception" as search keywords and setting search strategies, a total of 64 Chinese papers and 166 English papers were downloaded. In view of the earlier research on topics such as classroom learning and cheating in exams, and some of the downloaded papers contain mistrust of middle school students, it is necessary to screen the downloaded papers. The selection principles include: (1) Delete the repeatedly downloaded articles;(2) Check the language used in the paper; (3) Read the abstract and conclusions to ensure that the literature does not include those that are obviously beyond the scope contents; (4) The research scenario of the literature is an online learning environment or a digital learning environment, and no other learning scenarios are involved; (5) The introduction and text are also read. After the process was completed, the sample was reduced to 34 selected documents, all from 1997 to 2019 years, and the vast majority of the literature is about 10 years old.

\subsection{Conducting Phase}

A qualitative analysis of publications based on research questions was conducted to gain researchers' opinions or findings on online academic dishonesty. In order to explore the RQ1 of this study, the opinions of relevant literatures were comprehensively combed, and representative discussions were extracted. RQ2 is the focus of this research. Only by solving RQ2 can the formation mechanism of students' online academic dishonesty behavior, so this part has a more detailed analysis. In addition, RQ3 and RQ4 focus on the identification and intervention of online academic dishonesty, considering the background of educational big data and the development of smart learning in the future, so more choices for the 
research results of the past 5 years.

The limitations of this literature review include that the range of database selection is not wide, the criteria for literature selection are still subjective, the number of papers finalized is not enough, and only the literatures before 2019 are considered, which may result in some important literatures not being included, it limits the promotion value of the research conclusions.

\section{RESULTS}

RQ1: What are the characteristics and patterns of college students' learning dishonesty behavior in online learning?

At the level of modeling of dishonesty behavior, Blau et al. (2017) analyzed cheating behaviors in digital environment and non-digital environment, and found that although students believed that dishonesty was unethical, they still had dishonest behavior [7]. Empirical research by scholars such as Sullivan (2016) shows that if not curbed, the persistence, prevalence and propensity of academic fraud will pose a challenge to the integrity of online education [8]. At present, scholars' concerns about learning dishonesty are not limited to online learning participation, learning anxiety and learning experience, but also extend to their professional development and social moral performance [9], tracking by Underwood (2013) studies have shown that unscrupulous learning behaviors of students while in school will affect their performance in the workplace [10].

RQ2: What are the factors that cause college students to learn dishonesty in online learning?

At the level of attribution of dishonesty, previous studies have mostly attributed online academic dishonesty to technical aspects and personal factors (personality, cognitive ability, motivation, etc.). From the perspective of technology environment proponents, technology is the main means to promote academic fraud [11], but the research results of Sullivan et al. (2016) believe that digital technology is a channel for academic fraud, and the use of technology itself is not can significantly affect academic fraud [8]. From the perspective of personal motivation proponents, learners' attitudes, morals, and environmental perceptions are the underlying reasons for stimulating dishonesty. For example, Murdock and Anderman (2006) described the motivation model for academic dishonesty and summarized promoting factors and inhibiting factors of academic dishonesty [12]. Simkin et al. (2010) use rational behavior theory to build research models, use motivation, threats, class demonstrations to observe students' cheating attitudes and subjective norms, and analyze the relationship and cheating behavior intentions [13]. Cronan et al. (2015) introduced moral obligations and past experience (such as in high school) into the TPB model to analyze college students behavior of test plagiarism in online learning environment [14]. Anderman et al. (2017) empirical research shows perceptions of personal and class goals, self-efficacy, and consequences of cheating cognition can effectively reflect students 'intentions of dishonesty [15]. Stogner et al. (2013) research on criminology based on Akers (2009) shows that social learning variables (definition, differential association, imitation, and social reinforcement of different expectations) have a strong predictive effect on Internet cheating intentions and behavior frequency, but self-control and stress variables have little predictive effect [11]. Mcgee (2013) researches social support to promote academic performance, and proposed that teacher supervision and social activities in online courses lack can make students feel lonely, leading to a lack of awareness of their progress or achievements during the course [16].

RQ3: What are the methods to identify college students' learning dishonesty behavior in online learning?

At the level of dishonest behavior recognition, in the early days, researchers mostly used students' self-reporting methods for research. For example, Bashir et al. (2018) developed a scale that is suitable for detecting various types of learning dishonesty [17]. Peer et al. (2014) have found that students' reluctance to admit cheating means that self-reporting often underestimates academic dishonesty [18]. Later, researchers began to use a combination of self-reporting and computer algorithms to identify performance of dishonesty. For example, Baille and Jortberg (2009) argue that the identification and detection of plagiarism trails through technical means may be the key to limiting academic fraud [19]. Daradoumis et al. (2013) analyzed that the intelligent agent's reminder mechanism and automated evaluation method can effectively evaluate and monitor the learning process and learning performance of learners, enhance user authentication, and reduce fraud and cheating in exams [20]. Northcutt (2016) and Chuang (2017) have developed a dishonest behavior detection algorithm based on IP recognition [21] [22]. This is an effective means of identifying false learners in online learning and a prerequisite for behavioral intervention. Ruiperez-Valiente et al. (2019) applied machine learning algorithms to successfully detect "multi-account" cheating behavior, and analyzed the relationship between student personality characteristics and cheating behavior [23]. In addition, some researchers have tried to use learners' biometrics and behavior patterns to identify students, thereby preventing them from learning dishonesty [24] [25].

RQ4: What strategies can be adopted to correct or reduce the occurrence of college students' learning dishonesty?

At the level of dishonesty intervention, researchers have mainly adopted two methods: learning support and reward and punishment mechanisms. In terms of learning support, researchers emphasize the importance of teaching elements such as teacher presence, instructional design (time requirements, problem difficulty, etc.) to the online learning experience. For example, Paechter et al. (2010) believe that the importance of teachers has not decreased in e-learning, and students experience that teachers' support and professional knowledge are particularly important for them to acquire knowledge, skills and abilities, and curriculum satisfaction[26]. Mcgee et al. 
(2013) believe that promoting ethical behavior culture, changing evaluation strategies, and enhancing learners' self-awareness can promote students to stay away from online learning [16]. In the reward and punishment mechanism, honor and punishment mechanisms are also applied in teaching. Researchers try to realize the deterrence of students with dishonesty by reminding the consequences of behavior. For example, Freiburger et al. (2016) surveyed U.S. college students and found that teachers mentioned penalty provisions for dishonesty in the syllabus to increase the certainty of punishment, but this did not have a significant impact on dishonest behavior [27]. Corrigan-Gibbs et al. (2015) survey of Indian students shows that the use of honor badges in massive open online courses (MOOC) has a very limited effect on learning dishonesty, however, punishment has a more deterrent effect, and contains a wealth of warning information effects, which can reduce the occurrence of learning distrust behavior [28].

Compared with existing studies abroad, Chinese scholars have paid attention to the study of dishonesty in class, but there are few studies on online academic dishonesty. Most of the related researches are investigation and tracking of learning dishonesty and coping with common problems. At the level of attribution of dishonesty, a survey of 463 college students by Yang et al. (2013), a scholar in Taiwan, found that the three motivations of students' opportunism, lack of cognition, and self-improvement expectations have positive predictive effects on learning dishonesty [29]. Chen et al. (2018) analyzed the predictive effect of untrustworthy attitudes, subjective norms, perceived behavior control, morality and past dishonest experiences on the intention of dishonest behaviors in online learning based on extended planned behavior theory [30]. Hu's team (2016) put forward the concept of ethics of education virtual community, and studied the influencing factors of ethics of education virtual community and its interactive influence strategies on the community [31]. Relative to the study of learning dishonesty, domestic researchers have more research on network e-learning behavior. For example, Peng (2014) researched the connotation and triggers of misconduct behaviors in online environment, and found that misconduct behaviors have a greater impact on the quality and efficiency of e-Learning, but stimulate and maintain, negative emotions through motivation. regulation, reinforcement and active behavior support, learning strategy training and other strategies can improve and correct misfeasance [32]. At the level of dishonest behavior recognition, Liu (2019) analyzed the path of feature recognition and clustering algorithms to intelligently identify the anomie behavior of learners [33]. At the level of intervention in dishonesty behavior, Yang et al. (2013) surveyed college students in mainland China and Taiwan that a comprehensive education of academic integrity, improving students 'awareness of academic integrity and punishment, can effectively reduce the occurrence of students' dishonesty, and he also proposed the use of detection tools and learning services [29][34]. In summary, researchers in mainland China pay less attention to online academic dishonesty.

\section{CONCLUSIONS}

Online academic dishonesty is a typical problem learning behavior, which contains low immersion and poor experience of learning phenomena. Researchers have more research on the problem of learning dishonesty, which provides sufficient empirical support for subsequent research. Previous studies focus on the four aspects of academic dishonesty: characterization modeling, behavioral attribution, identification methods and intervention strategies.

Judging from the current development trend of college online courses, online learning as an autonomous learning mode without teacher supervision, will be more widely used in teaching reform. However, most of the existing studies abroad have explored the undergraduate's online learning behavioral characterization from explicit behaviors to infer behavior influencing factors, but in fact, the quantitative characteristics of explicit behaviors (number of plagiarism assignments, number of test exchange answers, etc.) cannot be directly reflect the internal motivation of the student. Although the scholars of Anderman et al mentioned the use of content analysis to analyze the attribution of academic dishonesty, it has not yet involved the analysis of cognitive-related thinking patterns. Especially in the SPOC environment and the mixed teaching environment, teachers and managers only discuss the undergraduate's online learning trust behavior through observation and student self-reporting, which may not necessarily infer its thinking mode and behavior motivation, which is exactly the future of intelligent learning individual learning characteristics that teachers and managers want to understand in an environment. On the basis of existing research, the combination of social learning theory and learning behavior analysis (LSA) method is used to explain the relationship between learner self-efficacy, social support, learning process management and learning dishonesty, which may form the mechanism of online academic dishonesty and intervention mechanisms produce more understandable research conclusions. It is worth noting that in developing countries, such as China, although there is a small amount of literature focusing on the study of online academic dishonesty, these studies remain at the stage of phenomenon observation and theoretical discussion. The research perspective is relatively narrow and the research depth is still not enough. The phenomenon of insufficient research on online academic dishonesty is not compatible with the increasingly in-depth application of online learning, which not only seriously affects the effectiveness of the construction of online courses in universities and the fundamental goal of building people, but also restricts the quality of education informatization in developing countries, and the modernization of education.

This study believes that future research requires interdisciplinary research on the mechanisms and prevention mechanisms of learners' online academic dishonesty under the network environment in universities, in combination with the theoretical resources of pedagogy, psychology, sociology, and information science. Based on 
the national conditions, learn from foreign research experience, and dig deep into the formation of online academic dishonesty and the operating rules and principles between the elements. Combining the three horizons of analysis with personality characteristics, learning environment and external reference, not only makes up for the concern about personality characteristics in previous research, but does not pay attention to the learning environment or external reference; Deepen the theoretical research on the formation mechanism and intervention mechanism of learners' online academic dishonesty behavior in the SPOC environment of colleges, and enrich the research results in this field. In addition, the problem of online learning integrity is related to the formation of college students' outlook on life and values. The conclusion of this study is also of certain reference value to ideological and political education in colleges. Future research should address these deficiencies in a targeted manner to better guarantee the quality of future online learning and the identification of achievements.

\section{ACKNOWLEDGEMENT}

The work was financially supported by the project of Shandong province higher educational research program(J18RA144), special project of Shandong Youth University of Political Science (XXPY18064, JG201814), research project of Shandong Youth University of Political Science (2015XGY05, SQ2016QN01), undergraduate teaching reform research project of Shandong province undergraduate teaching reform research project (Z2016M102), project of Shandong province higher educational science and technology program (J15LN15).

\section{REFERENCES}

[1] Pavela, Gary. "Applying the power of association on campus: A model code of academic integrity." JC \& UL 24 (1997): 97.

[2] King, Chula G., Roger W. Guyette Jr, and Chris Piotrowski. "Online exams and cheating: An empirical analysis of business students' views." Journal of Educators Online 6.1 (2009): 1-11.

[3] Raines, Deborah A., et al. "Cheating in online courses: The student definition." Journal of Effective Teaching 11.1 (2011): 80-89.

[4] Smole, David P., et al. "The higher education opportunity act: Reauthorization of the higher education act." Library of Congress, Congressional Research Service, 2008.
[5] Kocdar, Serpil, and Cengiz Hakan Aydin. "Quality assurance and accreditation of MOOCs: Current issues and future trends." Proceedings of Open Education Global (2015).

[6] Kitchenham, Barbara. "Procedures for performing systematic reviews." Keele, UK, Keele University 33.2004 (2004): 1-26.

[7] Blau, Ina, Yoram Eshet-Alkalai, and Adi Friedman. "Close educational gap by cheating? Comparing faculty, majority and minority students' perceptions of academic dishonesty." EdMedia+ Innovate Learning. Association for the Advancement of Computing in Education (AACE), 2017.

[8] Sullivan, Daniel P. "An Integrated Approach to Preempt Cheating on Asynchronous, Objective, Online Assessments in Graduate Business Classes." Online Learning 20.3 (2016): 195-209.

[9] McCabe, Donald L., Kenneth D. Butterfield, and Linda Klebe Trevino. "Academic dishonesty in graduate business programs: Prevalence, causes, and proposed action." Academy of Management Learning \& Education 5.3 (2006): 294-305.

[10] Underwood, Jean, and Attila Szabo. "Academic offences and e-learning: individual propensities in cheating." British Journal of Educational Technology 34.4 (2003): 467-477.

[11] Stogner, John M., Bryan Lee Miller, and Catherine D. Marcum. "Learning to e-cheat: A criminological test of internet facilitated academic cheating." Journal of Criminal Justice Education 24.2 (2013): 175-199.

[12] Murdock, Tamera B., and Eric M. Anderman. "Motivational perspectives on student cheating: Toward an integrated model of academic dishonesty." Educational psychologist 41.3 (2006): 129-145.

[13] Simkin, Mark G., and Alexander McLeod. "Why do college students cheat?" Journal of Business Ethics 94.3 (2010): 441-453.

[14] Cronan, Timothy Paul, Jeffrey K. Mullins, and David E. Douglas. "Further understanding factors that explain freshman business students' academic integrity intention and behavior: Plagiarism and sharing homework." Journal of Business Ethics 147.1 (2015): 197-220.

[15] Anderman, Eric M., and Alison C. Koenka. "The relation between academic motivation and cheating." Theory Into Practice 56.2 (2017): 95-102. 
[16] McGee, Patricia. "Supporting academic honesty in online courses." Journal of Educators Online 10.1 (2013): 1-31.

[17] Bashir, Hilal, and Ranjan Bala. "Development and validation of academic dishonesty scale (ADS): Presenting a multidimensional scale." International Journal of Instruction 11.2 (2018): 57-74.

[18] Peer, Eyal, Alessandro Acquisti, and Shaul Shalvi. "'I cheated, but only a little": Partial confessions to unethical behavior." Journal of Personality and Social Psychology 106.2 (2014): 202.

[19] Bailie, Jeffrey L., and Michael A. Jortberg. "Online learner authentication: Verifying the identity of online users." Journal of Online Learning and Teaching 5.2 (2009): 197-207.

[20] Daradoumis, Thanasis, et al. "A review on massive e-learning (MOOC) design, delivery and assessment." 2013 eighth international conference on P2P, parallel, grid, cloud and internet computing. IEEE, 2013.

[21] Northcutt, Curtis G., Andrew D. Ho, and Isaac L. Chuang. "Detecting and preventing "multiple-account" cheating in massive open online courses." Computers \& Education 100 (2016): 71-80.

[22] Chuang, Chia Yuan, Scotty D. Craig, and John Femiani. "Detecting probable cheating during online assessments based on time delay and head pose." Higher Education Research \& Development 36.6 (2017): 1123-1137.

[23] Ruiperez-Valiente, Jose A., et al. "Using machine learning to detect 'multiple-account'cheating and analyze the influence of student and problem features." IEEE Transactions on Learning Technologies 12.1 (2017): 112-122.

[24] Harmon, Oskar R., James Lambrinos, and Judy Buffolino. "Assessment design and cheating risk in online instruction." Online Journal of Distance Learning Administration 13.3 (2010).

[25] Kraglund-Gauthier, Wendy L., and David C. Young. "Chapter 17 Will the Real "John Doe" Stand up? Verifying the Identity of Online Students'." Misbehavior Online in Higher Education (Cutting-Edge Technologies in Higher Education, Volume 5). Emerald Group Publishing Limited (2012): 355-377.

[26] Paechter, Manuela, Brigitte Maier, and Daniel Macher. "Students' expectations of, and experiences in e-learning: Their relation to learning achievements and course satisfaction." Computers \& education 54.1 (2010): 222-229.

[27] Freiburger, Tina L., et al. "Cheating behaviors among undergraduate college students: Results from a factorial survey." Journal of Criminal Justice Education 28.2 (2017): 222-247.

[28] Corrigan-Gibbs, Henry, et al. "Deterring cheating in online environments." ACM Transactions on Computer-Human Interaction (TOCHI) 22.6 (2015): 1-23.

[29] Yang, Shu Ching, Chiao-Ling Huang, and An-Sing Chen. "An investigation of college students' perceptions of academic dishonesty, reasons for dishonesty, achievement goals, and willingness to report dishonest behavior." Ethics \& Behavior 23.6 (2013): 501-522.

[30] Chen,Changsheng, Xiangzeng Meng, ZhenGuo Xu, Zhi Liu, and JunXiao Liu.Study on influencing factors of college students' intention of dishonest behaviors in e-learning. Modern Distance Education 6 (2018): 3-12.

[31] Hu,fanggang, Wei Liu, ZhiYuan Meng, and YongQi Liu. "Empirical analysis of factors influencing ethical anomy in virtual communities of education." research on audio-visual education 37.3 (2016): 26-33.

[32] Peng wenhui." study on learning behavior in network problems." China Educational Technology 2.325 (2014): 40-45.

[33] Liu, Shutang, Fuquan Zhang, and Chunfen Jiang. "Preliminary Ideas for Intelligent Recognition Paths of Anomie Behaviors Among MOOC Learners." International Conference on Smart Vehicular Technology, Transportation, Communication and Applications. Springer, Cham, 2018.

[34] Yang, Shu Ching, Feng Kuang Chiang, and Chiao Ling Huang. "A comparative study of academic dishonesty among university students in Mainland China and Taiwan." Asia Pacific Education Review 18.3 (2017): 385-399. 